\title{
Low Cost Device for Precise Microliter Range Liquid Dispensing
}

\author{
N. Roxhed, S. Rydholm, B. Samel, W. van der Wijngaart, P. Griss ${ }^{1}$ and G. Stemme \\ Microsystem Technology, Department of Signals, Sensors and Systems, \\ Royal Institute of Technology, Stockholm, Sweden
}

\begin{abstract}
In this work we present the fabrication and testing of a thermally actuated one-shot liquid dispenser, which actuation is based on highly expandable microspheres. We show an uncomplicated, fully functional, low cost device for use in medical disposables, e.g. transdermal systems based on microneedles. All device components are made out of low cost materials and fabrication processes have the potential for high volume batch manufacturing. The device utilizes the properties of the expandable microspheres to form a heat insulating layer to the delivered liquid. Moreover, it does not require any feed-back or complicated flow metering.

The device was successfully tested showing a mean dispensed liquid of $101 \mu \mathrm{l}$ with a relative standard deviation of $3.2 \%$ and with a maximum temperature of $59^{\circ} \mathrm{C}$ in the liquid during actuation. No back-flow was observed for the device.
\end{abstract}

\section{INTRODUCTION}

There is a growing need for actuation means in the field of disposable medical systems. Intradermal injections, specifically, form a field of application that requires the actuation of liquid through the skin. Such applications range from treatment of dermatological diseases, e.g. localized chemotherapy, to vaccinations with macromolecular vaccines through the skin. To reduce costs and to avoid incorrect dosing it is important to precisely control the amount of drug delivered. Furthermore, it is favorable if drug delivery systems are easy operable and low cost, making disposable devices desirable.

Several groups have made liquid displacing actuators especially intended for medical drug delivery systems [1]-[3]. However, most of them focus on pump-like structures delivering continuous flows which require either extraordinary repeatability (feed-forward) or complicated flow metering (feed-back) for accurate delivery. Other groups have concentrated on making one-shot dispensing devices where dosing precision is geometrically defined [4]-[7]. However, precise, low cost, uncomplicated devices suitable for reasonable amounts of drugs used in medicine today are still lacking. In this work we have addressed such issues by making an uncomplicated low cost device actuated with expandable microspheres.

Previously our group suggested thermally exited expanding microspheres as a possible actuator for displacement of liquids. We demonstrated the use of expandable microspheres

\footnotetext{
${ }^{1}$ Presently with Roche Diagnostics Microtechnology Center, Rotkreuz, Switzerland
}

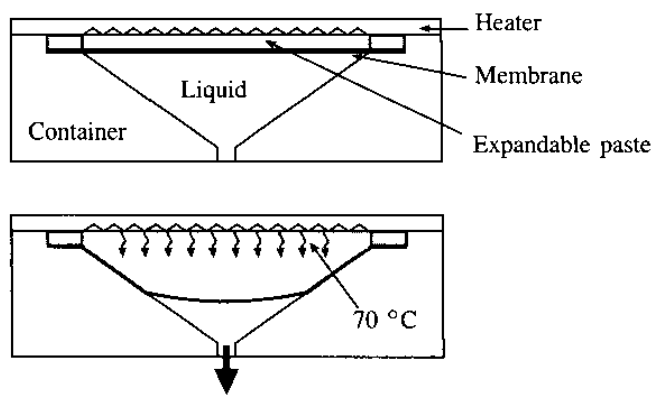

Figure 1: Schematic view of the working principle. As the expandable paste reaches $70{ }^{\circ} \mathrm{C}$ expansion will push the liquid out of the container. Throughout the expansion the heat conduction reduces and the paste acts as a thermal protection layer towards the liquid.

in microfabrication where we show bead expansion in a microchannel [8] and a novel solid compound by incorporating beads in PDMS [9]. When heated, the polymer shell of the gas filled microspheres softens and the volume increases up to 60 times its original volume (fig. 2).

\section{DESIGN}

In this work we present a thermally actuated liquid dispenser intended for use in conjunction with microneedles and transdermal drug delivery systems. The dispenser is designed for one-shot usage and made to meet requirements of disposable drug delivery system. Hence, all device components are made out of low cost materials and adapted for processes with the potential for high volume batch fabrication. The dispensing actuator uses highly expandable microspheres (Expancel ${ }^{\circledR}$ Microspheres) for its actuation. The device presented in this work utilizes the thermodynamical properties of the expandable microspheres to form a heat insulating layer towards the delivered liquid and thereby preventing extensive heating of the liquid.

The principle of operation is shown in figure 1; a small portion of an expandable paste containing Expancel ${ }^{\circledast}$ microspheres and Glycerin is heated from one side. When the paste reaches a temperature of $70^{\circ} \mathrm{C}$ it gradually expands into the liquid container. The liquid, separated from the paste by a thin elastic membrane, is pushed out as the expanded paste fills the whole container. Since volume expansion of the paste is manyfold, the heat conduction in the paste gradually drops to a considerably lower level, preventing the dispensed liquid from extensive heating. This protects possible temperature 


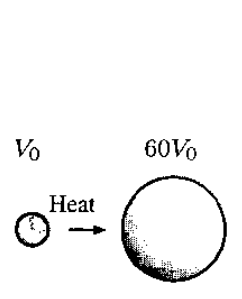

(a)

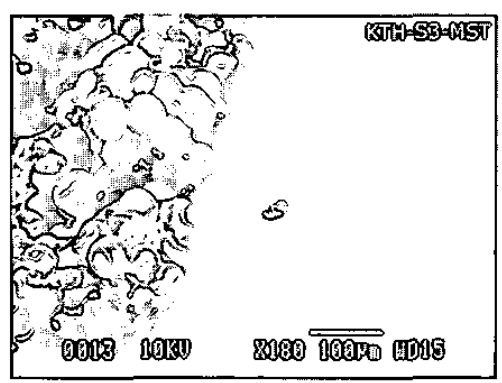

(b)
Figure 2: Expancel ${ }^{\circledR}$ Microspheres are gas filled polymer spheres: (a) Single spheres expands up to 60 times when heated. Initial diameter is typically $10 \mu \mathrm{m}$. (b) SEM picture showing expanded microspheres.

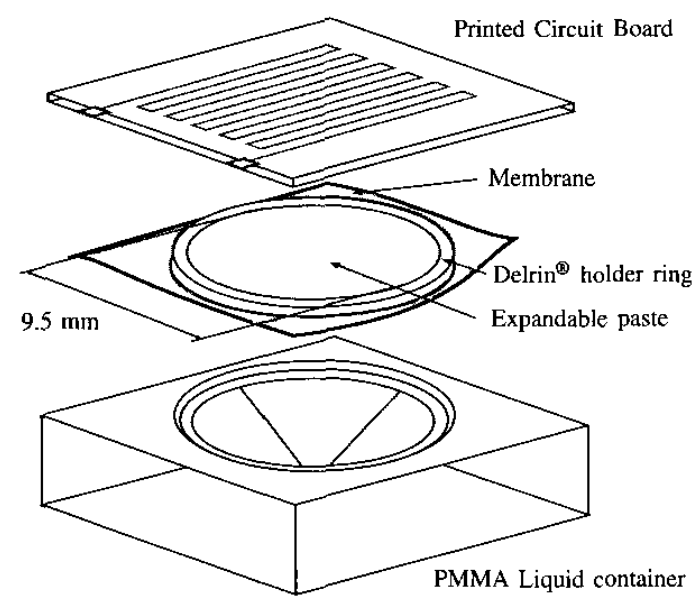

Figure 3: Schematic view of the device. The ring with the expandable paste together with the membrane is clamped into the liquid container, thereby stretching the membrane to become flat.

sensitive liquids such as drugs. The expansion is irreversible and therefore no back-flow occurs, hence valve structures are not needed.

\section{Fabrication}

The structure (fig. 3) consists of a $100 \mu \mathrm{l}$ liquid container made of a cone shaped cavity in PMMA (Polymethylmethacrylate). This building block is combined with a $0.5 \mathrm{~mm}$ high, Delrin ${ }^{\text {(3) }}$ made, holder ring encircling $35 \mu \mathrm{l}$ of unexpanded paste. The ring and the paste are isolated by a $200 \mu \mathrm{m}$ thick elastic vinyl membrane to chemically separate the expanding material from the delivery liquid. The membrane and the ring are clamped into the liquid container and a conventional printed circuit board (PCB) with a photolithographically defined copper heater is attached to the expandable medium on the open side of the ring. The assembled device is filled with water by submerging the fully

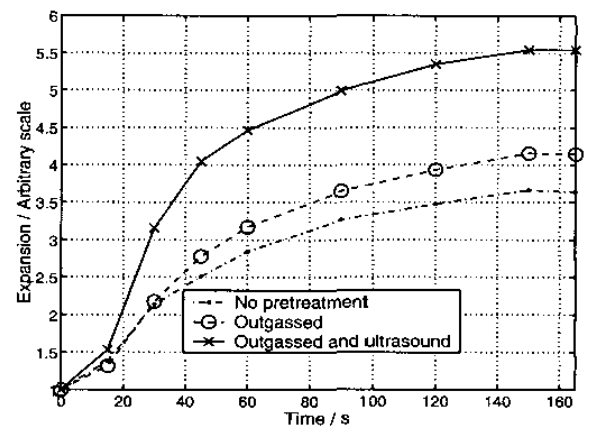

Figure 4: Different pretreatments show a significant variation in the level of expansion for expandable microspheres mixed in DI-water. Pretreatment with ultrasound showed a considerable expansion increase [10].

assembled device in water and applying vacuum.

The properties of expandable microspheres mixed in a liquid have been studied and measurements show that the pretreatment of the microsphere solution has a significant influence on the expansion level [10]. For microsphere/DIwater solutions it was found that an outgassed solution treated with ultrasound gives the largest expansion, see figure 4 . This study also showed that a moderate concentration $(0.3 \mathrm{~g} / \mathrm{ml})$ of expandable microspheres gives just as good or even slightly better expansion than a highly concentrated solution. However, thermodynamical effects were not addressed in this study.

In this work the expandable paste is made by mixing Expancel 820DU microspheres and Glycerin. Glycerin is chosen as the microsphere intermediate and mixing agent because it is a fairly good heat conductor and it has a high boiling point, which prevents evaporation. A concentration of Expancel microspheres of $0.7 \mathrm{~g} / \mathrm{ml}$ Glycerin is used to create a sufficiently moisturized, and heat conducting, paste with a suitable consistency for screen printing. The paste is outgassed for $10 \mathrm{~min}$. in vacuum and treated with ultrasound for $15 \mathrm{~min}$.

\section{EXPERIMENTAL}

The device was tested with respect to precision in liquid delivery and maximum temperature of the liquid during actuation. Precision was measured by connecting a fully assembled device to glass capillary. Interconnecting silicone tubings and part of the glass capillary were filled with water. As the liquid dispenser was actuated the displacement of the water meniscus in the capillary was measured with a read off error of approximately $0.39 \mu \mathrm{l}$.

In order to evaluate the effectiveness of the expanding material as an active heat insulator and monitor the heat dissipation to the liquid a temperature sensor was placed inside the liquid container. A small SMD NTC-thermistor (Mitsubishi TN10) was used to locally measure the temperature in the center of the structure at the liquid-membrane interface where the highest temperature occurs. 


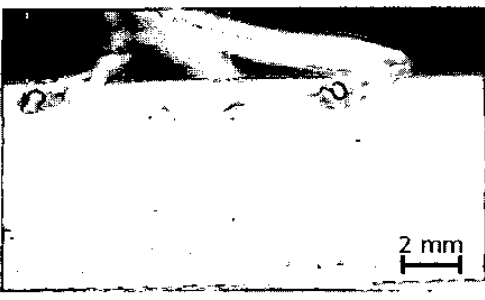

(a) Unexpanded (state before actuation).

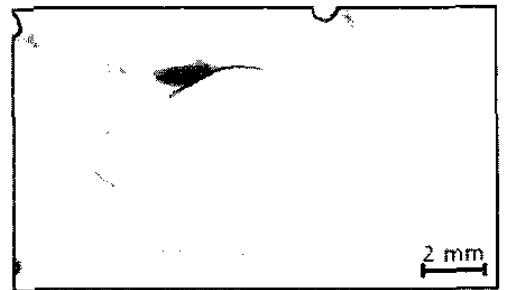

(b) Expanded (state after actuation; liquid container removed for visualization).

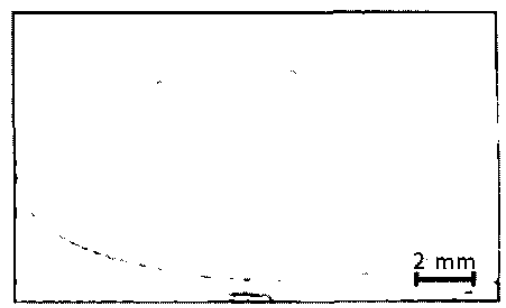

(c) Expansion without expansion-constraining liquid container results in a volume expansion of seven times the original volume.

Figure 7: Photographs of the expandable paste on the PCB-heater. The initial volume (a) is $35 \mu l$.

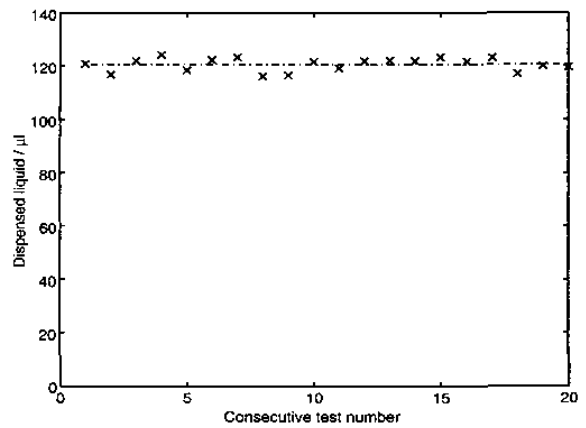

Figure 5: The device was tested with water as the dispensed liquid. 20 consecutive tests with successive reassemble were performed showing a standard deviation of $3.2 \%$.

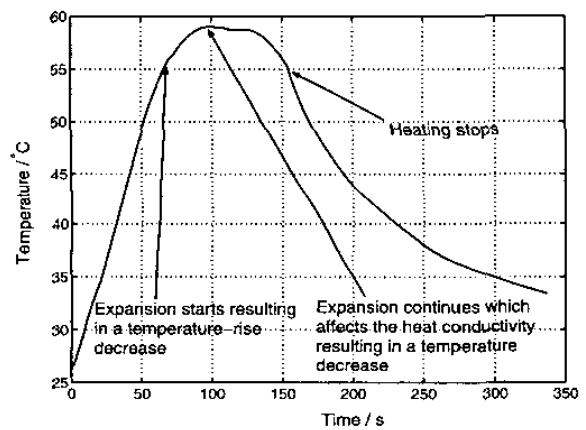

Figure 6: Temperature in the dispensed liquid during actuation. The temperature is measured at the membrane interface in the center of the structure.

\section{RESULTS AND DISCUSSION}

Precision measurements were conducted by applying a mean power of $1 \mathrm{~W}$ during $150 \mathrm{~s}$ to the PCB-heater of the device. 20 consecutive measurements with successive reassembly were made showing a mean dispensed liquid of $101 \mu \mathrm{l}$ with a relative standard deviation of $3.2 \%$ (fig. 5). Deviations in these measurement are believed to mainly origin from the manual reassembly of the device. Possible error sources include proper refilling, displacements of individual parts and the flatness of the separating vinyl membrane. It is likely to believe that these effects can be reduced by automated assembly.

The temperature in the liquid container during actuation was measured under the same heating condition as described above. The temperature profile throughout the actuation is shown in figure 6 . The measurement shows that during a short amount of time the temperature peaks at $59{ }^{\circ} \mathrm{C}$. From figure 6 it can clearly be seen that after approximately $70 \mathrm{~s}$ the temperature rise decreases. This corresponds to the moment that the expandable paste starts to expand (i.e. when the paste is heated to $70^{\circ} \mathrm{C}$, starting from room temperature). As the expansion continues the expanding material becomes an efficient heat insulator. This results in temperature decrease in the liquid, although the device is still being actuated and heated. Note that the actual dispensing only takes place when the paste is expanding, i.e. during the last $80 \mathrm{~s}$. This means that controlling the power distribution in time can substantially reduce the dispensing time.

Photographs of the different states of the actuator are shown in figure $7 \mathrm{a}-\mathrm{b}$. Figure $7 \mathrm{c}$ shows the expanded paste when no geometry constraining liquid container was used, demonstrating the potential of the expandable microspheres.

\section{Conclusions and outlook}

We introduced a thermally actuated one-shot liquid dispenser made of low cost materials and adapted for inexpensive batch fabrication.

The device was successfully tested showing a mean dispensed liquid of $101 \mu \mathrm{l}$ with a relative standard deviation of $3.2 \%$ and with a maximum temperature of $59{ }^{\circ} \mathrm{C}$ in the liquid. No back-flow was observed during the evaluation of the device.

Variation in the measurements is believed to mainly origin from the manual assembly of the device and improvement through batch fabrication is likely. Moreover, it was measured that pretreatment of the expandable microspheres has a large influence on the amount of energy needed for actuation. The properties of the expandable microspheres can be tuned to decrease expansion temperature. The present design leaves enough room for improvements to increase delivery pre- 
cision, to reduce required actuation energy, to interface it to microneedle chips, and to adapt the device to thermal requirements of liquids (e.g. drugs).

\section{REFERENCES}

[1] S. Bohm, W. Olthuis, and P. Bergveld, "An integrated micromachined electrochemical pump and dosing system," Biomedical Microdevices, vol. 1, no. 2, pp. 121130, 1999.

[2] M. Richter, N. Lutter, A. Leukert, U. Schaber, M. Wackerle, E. Kozma, and K. Neuder, "A portable drug delivery system with silicon capillaries as key components," in Proceedings of 11th International Conference on Solid State Sensors and Actuators Transducers 'OI/Eurosensors XV, 10-14 June 2001, pp. 970-973.

[3] D. Maillefer, S. Gamper, B. Frehner, P. Balmer, H. van Lintel, and P. Renaud, "A high-performance silicon micropump for disposable drug delivery systems," in Technical Digest. MEMS 2001. 14th IEEE International Conference on Micro Electro Mechanical Systems, 21 25 Jan. 2001, pp. 413-417.

[4] L. Guerin, O. Dubochet, J.-F. Zeberli, P. Clot, and P. Renaud, "Miniature one-shot valve," in Proceedings IEEE Eleventh Annual International Workshop on Micro Electro Mechanical Systems An Investigation of Micro Structures, Sensors, Actuators, Machines and Systems, 25-29 Jan. 1998, pp. 425-428.

[5] R. Scheidt, J. Santini Jr., A. Richards, A. Johnson, A. Rosenberg, M. Cima, and R. Langer, "Microchips as implantable drug delivery devices," in Ist Annual International IEEE-EMBS Special Topic Conference on Microtechnologies in Medicine and Biology. Proceedings, 12-14 Oct. 2000, pp. 483-486.

[6] S. Koster, A. Blau, C. Ziegler, J.-C. Roulet, N. F. de Rooij, and E. Verpoorte, "Dispensing system for localized stimulation of neural cell networks," in Proceedings of $\mu$ TAS 2003 Seventh International Conference on Micro Total Analysis Systems, pp. 93-96.

[7] A. Puntambekar, C. Hong, C. Gao, X. Zhu, R. Trichur, J. Han, S. Lee, J. Kai, J. Do, R. Rong, S. Chilukuru, M. Dutta, L. Ramasamy, S. Murugesan, R. Cole, J. Nevin, G. Beaucage, J. Lee, J. Lee, M. Bissellj, J. Choi, and C. H. Ahn, "Smart disposable plastic labon-a-chip for point-of-care testing (poct)," in Proceedings of $\mu$ TAS 2003 Seventh International Conference on Micro Total Analysis Systems, pp. 1291-1294.

[8] P. Griss, H. Andersson, and G. Stemme, "Liquid handling using expandable microspheres," in Technical Digest. MEMS 2002 IEEE International Conference. Fifteenth IEEE International Conference on Micro Electro Mechanical Systems, 20-24 Jan. 2002, pp. 117-120.

[9] B. Samel, P. Griss, and G. Stemme, "Expandable microspheres incorporated in a pdms matrix: A novel thermal composite actuator for liquid handling in microfluidic applications," in Transducers'03 The 12th International Conference on Solid-State Sensors, Actuators and Microsystems, pp. 1558-1561.
[10] S. Rydholm, "Expandable microspheres for liquid actuation and dosing," Master's thesis, Royal Institute of Technology, Dept. of Signals, Sensors and Systems, March 2003. 